\title{
ORIGINAL ARTICLE \\ Cardiorespiratory responses during functional electrical stimulation cycling and electrical stimulation isometric exercise
}

\author{
C Fornusek ${ }^{1}$, TH Gwinn ${ }^{1}$ and $\mathrm{R}$ Heard $^{2}$
}

Study design: Prospective experimental.

Objectives: To compare the cardiorespiratory responses with electrical stimulation (ES) producing either dynamic leg cycling or intermittent isometric leg contractions using the same ES protocol.

Setting: Sydney, Australia.

Methods: Eight paraplegics (T4-T11) performed ES exercise sessions on two separate days. On day 1, cardiorespiratory responses were measured during $5 \mathrm{~min}$ of rest followed by $35 \mathrm{~min}$ of cycling, and finally $15 \mathrm{~min}$ of intermittent isometric exercise using the same ES parameters. On the second day, after $5 \mathrm{~min}$ of rest, $35 \mathrm{~min}$ of isometric exercise was performed followed by $15 \mathrm{~min}$ of cycling.

Results: There were no significant differences during the first $35 \mathrm{~min}$ of exercise on each day comparing the two modes of exercise for average rate of oxygen consumption (cycling, $534 \pm 128 \mathrm{ml} \mathrm{min}^{-1}$; isometric $558 \pm 146 \mathrm{ml} \mathrm{min}^{-1} ; P=0.451$ ), the average heart rate (cycling, $93 \pm 15$ b.p.m.; isometric $95 \pm 17$ b.p.m.; $P=0.264$ ) or minute ventilation (cycling, $23.0 \pm 6.5 \mathrm{Imin}-1$; isometric $\left.23.8 \pm 6.71 \mathrm{~min}^{-1} ; \quad P=0.655\right)$. In addition, there were no significant differences between exercise modes for any peak cardiorespiratory values recorded during the initial $35 \mathrm{~min}$ of exercise or the following 15 min crossover exercise phase.

Conclusion: The current data found that intermittent ES leg isometric exercise elicited a similar cardiorespiratory response compared with functional ES leg cycling, suggesting it should be investigated as a viable alternative intervention for increasing whole body metabolic rate during sustained exercise training sessions for individuals with paralyzed muscles.

Spinal Cord (2014) 52, 635-639; doi:10.1038/sc.2014.85; published online 3 June 2014

\section{INTRODUCTION}

The promotion of life-long engagement in regular physical activity is important within the spinal cord injury (SCI) population to improve health, fitness and quality of life. ${ }^{1}$ Aerobic training recommendations for those with SCI are similar to guidelines for the general population. ${ }^{2}$ In line with such guidelines, it has been recommended that SCI individuals perform 3-5 exercise sessions per week of 20-60 min in duration at a moderate to high percentage of $\dot{V} \mathrm{O}_{\text {2peak. }}{ }^{2}$ More recent recommendations have suggested that minimum levels of two $20 \mathrm{~min}$ sessions per week are more realistic and sustainable for people with SCI. ${ }^{1}$

Although individuals with paraplegia, and to a lesser extent some individuals with tetraplegia, can perform arm exercise training, as the arm muscle mass represents a relatively small percentage of total body muscle mass, the absolute $\dot{V}_{\mathrm{O}_{2}}$ at which they can exercise is generally limited as compared with the able-bodied individuals. An alternative is via the use of electrical stimulation (ES) to paralyzed lower limb muscle groups. One common form of ES to produce whole body elevations in $\dot{V}_{\mathrm{O}_{2}}$ in SCI is functional ES cycling (FES), with electrically activated contractions of the quadriceps, hamstrings and gluteus muscles under computer microprocessor command. ${ }^{2}$ Some authors use the term FES to describe this type of dynamic exercise.
One goal of FES research has been to create exercise systems that will elicit an oxygen consumption $\left(\dot{V}_{\mathrm{O}_{2}}\right)$ in SCI sufficient to obtain health benefits with repeated training bouts. ${ }^{3}$

Until recently, the use of stimulated isometric contractions has received little attention as an alternative to FES dynamic contractions as a practical way for individuals with SCI to perform 'aerobic' exercise training. One reason for this may be the impression that isometric contractions, in general, are associated with a relatively low metabolic cost compared with concentric contractions; an effect first described by Fenn ${ }^{4}$ in 1925 as the 'extra heat of shortening'. However, it is important to note that these results are specific to prolonged, continuous isometric contractions. In contrast, for example, Newham et al. ${ }^{5}$ found cyclic dynamic contractions and intermittent isometric contractions of the human adductor pollicis, elicited with the same ES protocol, to have a similar energy cost, with both these modes of exercise having significantly higher energy costs compared with a continuous isometric contraction.

We know of no studies that have compared the energy cost associated with dynamic loading in FES cycling with isometric loading using the identical stimulation protocol in SCI subjects. Our hypothesis was that despite the marked differences in the extent of limb movement, the energy cost and associated

${ }^{1}$ Exercise Health and Performance Research Group, Faculty of Health Sciences, University of Sydney, Sydney, Australia and ${ }^{2}$ Discipline of Behavioural and Social Sciences in Health, Faculty of Health Sciences, University of Sydney, Sydney, Australia

Correspondence: Dr C Fornusek, Exercise Research and Performance Research Group, University of Sydney, Faculty of Health Sciences, 75 East Street, Sydney 2141, Australia. E-mail: che.fornusek@sydney.edu.au

Received 17 November 2013; revised 19 April 2014; accepted 3 May 2014; published online 3 June 2014 
Table 1 Participant characteristics

\begin{tabular}{lcccccc}
\hline Subject & $\begin{array}{c}\text { Age } \\
\text { (years) }\end{array}$ & Gender & $\begin{array}{c}\text { Weight } \\
(\mathrm{kg})\end{array}$ & $\begin{array}{c}\text { Level of } \\
\text { injury (A/S) }\end{array}$ & $\begin{array}{c}\text { Time since } \\
\text { injury (years) }\end{array}$ & $\begin{array}{c}\text { Training } \\
\text { status }\end{array}$ \\
\hline 1 & 43 & Male & 52 & T4 (A) & 18 & $3 x / w k, 24 \mathrm{mo}$ \\
2 & 28 & Male & 96 & T4 (A) & 2 & $3 x / w k, 24$ mo \\
3 & 63 & Male & 60 & T4 (A) & 10 & $2 x / w k, 6$ mo \\
4 & 55 & Male & 70 & T8 (A) & 16 & $2 x / w k, 6$ mo \\
5 & 60 & Male & 83 & T11 (C) & 12 & $2 x / w k, 3$ mo \\
6 & 32 & Male & 82 & T6 (A) & 3 & $2 x / w k, 1$ mo \\
7 & 64 & Male & 81 & T8 (A) & 8 & $2 x / w k, 1$ mo \\
8 & 39 & Male & 77 & T8 (A) & 2 & $3 x / w k, 6$ mo
\end{tabular}

Abbreviations: AIS, ASIA impairment scale; ASIA, American Spinal Injury Association;

C, cervical; mo, months; T, thoracic; wk, weeks.

cardiorespiratory responses would be similar in comparing the two forms of loading.

\section{MATERIALS AND METHODS}

\section{Participants}

Eight individuals with chronic paraplegia (T4-T11) participated in this study (Table 1). Participants were recruited as a sample of convenience from those attending the FitAbility clinic at the Discipline of Exercise and Sports Science. Participants were excluded if maximum stimulation elicited symptoms of autonomic dysreflexia. Seven of the participants had complete spinal cord injury (American Spinal Injury Association (ASIA) A) and one had an incomplete injury (ASIA C). The subject with incomplete injury could not produce joint movements against gravity, could comfortably tolerate the stimulation and was instructed not to push during these experiments. All participants could complete $35 \mathrm{~min}$ of FES cycling. The experimental protocol was approved by the Ethics Committee of the University of Sydney. All participants provided written informed consent.

\section{Testing protocol}

Each volunteer participated in two sessions that involved periods of FES cycling and ES isometric exercise (Figure 1). The test sessions were performed 1 week apart. The subjects were seated on a recumbent FES cycle ergometer (Figure 2). During the first session, three phases of exercises were performed: (1) $5 \mathrm{~min}$ of resting, (2) $35 \mathrm{~min}$ of FES cycling exercise at $50 \mathrm{rev} \mathrm{min}^{-1}$, and (3) 15 min of intermittent ES isometric exercise where the pedals were locked in a fixed position. For the second session, the order and durations of the ES isometric and FES cycling were swapped.

\section{Neuromuscular stimulation}

Dynamic cycling and isometric contraction used an identical stimulation protocol. The stimulation comprised biphasic rectangular pulses at a frequency of $35 \mathrm{~Hz}$ and pulse width of $300 \mu$ s. Stimulation was delivered via pairs of gelbacked surface electrodes (Empi, St Paul, MN, USA) placed on the quadriceps, hamstrings and glutei muscles. Electrode placement was kept consistent between sessions to ensure that muscle fiber recruitment was similar.

At the beginning of phase 2, stimulation amplitude was linearly ramped from 40 to $140 \mathrm{~mA}$ over $10 \mathrm{~min}$, and then held constant at $140 \mathrm{~mA}$ for the next $25 \mathrm{~min}$. As isokinetic ergometers were used, the stimulation intensity did not have to be modulated to maintain the pedaling cadence as is usual during FES cycling. During the transition from phase 2 to phase 3 (that is, FES cycling to isometric ES or vice versa), the stimulation was temporarily disabled while the cable that supplied pedal position to the laptop computer was switched from one cycle ergometer to the other. Once the transition was complete the stimulation pulse amplitude was rapidly restored to $140 \mathrm{~mA}$ and held constant for the remainder of the session.

\begin{tabular}{|l|c|c|c|}
\cline { 2 - 4 } \multicolumn{1}{c|}{} & $\begin{array}{c}\text { Phase 1 } \\
(5 \mathrm{~min})\end{array}$ & $\begin{array}{c}\text { Phase 2 } \\
(35 \mathrm{~min})\end{array}$ & $\begin{array}{c}\text { Phase 3 } \\
(15 \mathrm{~min})\end{array}$ \\
\hline Session 1 & Rest & ES cycling & Isometric ES \\
\hline Session 2 & Rest & Isometric ES & ES cycling \\
\hline
\end{tabular}

Figure 1 The order of tests performed during the two sessions that were separated by a week.

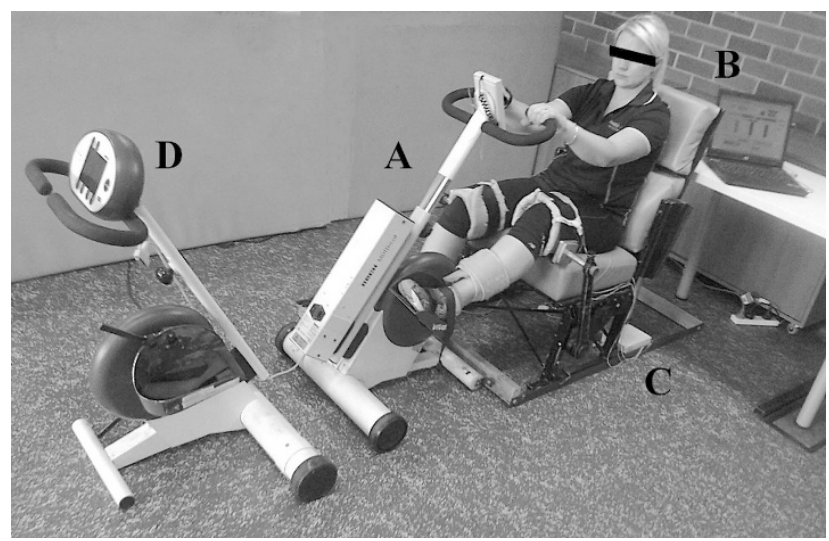

Figure 2 The setup used for the FES cycling and isometric ES. A, motomed ergometer and recumbent seating for FES cycling; B, control computer; C, muscle stimulator; D, second motomed ergometer for isometric stimulation timing.

\section{ES cycle ergometer}

A custom-designed stationary FES cycle ergometer was used (Figure 2). ${ }^{6}$ The components of the FES cycle ergometer were an isokinetic cycle ergometer (MOTOmed Viva, RECK-Technik GmbH, Betzenweiler, Germany), a muscle stimulator and a laptop running control software. The ergometer sent crank position and power data to the computer that initiated stimulation at the appropriate crank angles to drive the pedals. The laptop recorded the power output from the ergometer. Each subject's foot was fixed on the pedals using inextensible Velcro straps.

\section{ES isometric exercise}

ES isometric exercise occurred on the same FES cycle ergometer with the crank axle position fixed (horizontal and pointing forward). This resulted in isometric contractions with the right knee at $\sim 115^{\circ}$, a right thigh-trunk angle of $\sim 85^{\circ}$, the left knee at $\sim 70^{\circ}$ and a left thigh-trunk angle of $\sim 65^{\circ}$. During the ES isometric exercise, the stimulation was triggered from the pedal position of another Motomed Viva2 cycle ergometer whose pedals were rotating at $50 \mathrm{rev} \mathrm{min}^{-1}$. Therefore, the stimulation parameters delivered during the ES isometric exercise were identical to those applied during FES cycling including stimulation intensity, duration and rest interval to each muscle group.

\section{Recorded data}

Cardiorespiratory activity (oxygen consumption $\left[\dot{V}_{\mathrm{O}_{2}}\right]$, ventilation $\left[\dot{V}_{\mathrm{E}}\right]$, tidal volume $[\mathrm{VT}]$,$] ) was assessed at rest and during each exercise session$ continuously with an open-circuit metabolic gas analysis system (Ultima series, Medgraphics, St Paul, MN, USA). Heart rate (HR) was measured by a finger pulse oximeter (Nonin Model 7500, Plymouth, MN, USA) connected to the metabolic gas analysis system. The power output (W) produced during FES cycling was recorded. 


\section{Data analysis}

The last 2 min of resting (phase 1) cardiorespiratory data were averaged to give representative values of resting metabolism for each session. The cardiorespiratory data from each session were averaged every $15 \mathrm{~s}$ to give a representative data point every $15 \mathrm{~s}$. The peak values for each cardiorespiratory variable were then determined for both exercise phases of each session (for example, phase $2(0-35 \mathrm{~min})$ and phase $3(35-50 \mathrm{~min}))$. An average value was also obtained for each cardiorespiratory variable over phase 2 for each session (that is, $35 \mathrm{~min}$ of FES cycling or isometric ES). Data are reported as mean (s.d.).

\section{Statistical analysis}

Each of the cardiorespiratory measurements were analyzed using two-way (session $(\mathrm{S} 1, \mathrm{~S} 2) \times$ phase (phase 1 (rest), phase 2 (exercise $35 \mathrm{~min}$ ), phase 3 (exercise 35-50 $\mathrm{min})$ )) repeated-measures analysis of variance, carried out as two-factor planned orthogonal contrasts with repeated measures on both factors. The main research questions were whether intervention affected performance compared with rest (comparisons 1 and 2 below) and whether cycling differed in mean score from isometric (comparisons 3 and 4). Interactions that do not directly address these questions are not reported.

Data for each variable were analyzed using four main effect planned orthogonal contrasts: (1) rest (phase 1) versus later interventions (phases 2 and 3); (2) phase 2 versus phase 3; (3) session 1 phase 2 (cycling) versus session 2 phase 2 (isometric); and (4) session 1 phase 3 (isometric) versus session 2 phase 3 (cycling).

The average values of the cardiovascular measures were also analyzed using a two-way (session $(\mathrm{S} 1, \mathrm{~S} 2) \times$ phase (phase 1 (rest), phase 2 (exercise $35 \mathrm{~min})$ ) repeated-measures analysis of variance. Paired $t$-tests were used to compare between the average values attained over $35 \mathrm{~min}$ with FES cycling and ES isometric. In all cases, $P<0.05$ was deemed significant. Confidence intervals (80\% power; $\alpha=0.05$ ) were calculated for the mean differences elicited in cardiovascular responses from FES cycling and isometric ES. All statistical analyses were made using the SPSS V21 (Chicago, IL, USA).

We certify that all applicable institutional and governmental regulations concerning the ethical use of human volunteers were followed during the course of this research.

\section{RESULTS}

The average power during the last $10 \mathrm{~min}$ of FES cycling (that is, 25-35 min) during session 1 was $10.3 \pm 8.2 \mathrm{~W}$ (range 3.4-27.1 W). The power output from the $10 \mathrm{~min}$ of the FES cycling during session 2 was of a similar magnitude $(9.4 \pm 10.4 \mathrm{~W}$; range $2-33.5 \mathrm{~W})$. In contrast to FES cycling, the periods of ES isometric muscle contractions produced a very slight straightening of the knees, very little movement of the pedals or crank and zero power on the crank.

Statistical analysis indicated that there was an effect of phase. Both FES cycling and isometric ES induced significant increases (for all variables $P=0.001$ ) from rest values for all cardiorespiratory measures (Table 2). HR, oxygen consumption and ventilation all increased during the first exercise period (phase 2). HR increased slowly during each session until $\sim 30 \mathrm{~min}$ where it plateaued (Figure 3). In contrast, oxygen consumption and minute ventilation increased rapidly in the first $10-15 \mathrm{~min}$ but then plateaued until $\sim 25 \mathrm{~min}$, and then decreased slightly (Figures 4 and 5). No obvious changes in metabolism were present for any variable at $35 \mathrm{~min}$ when the switch occurred between exercise modes (that is, cycling to isometric and vice versa). However for the respiratory values $\left(\dot{V}_{\mathrm{O}_{2}}\right.$, $\dot{V}_{\mathrm{E}}$ and $\left.\mathrm{V}_{\mathrm{T}}\right)$ there was a significant decrease $(P=0.001$ for each) in the peak values achieved in phase 2 to phase 3 irrespective of type of exercise in the phases. HR did not differ between phase 2 and phase 3 $(P=0.285)$.

No significant differences were found between sessions 1 and 2, except for resting HR being slightly higher before session 1
$(P=0.015)$. The stimulation exercise mode used did not affect the cardiorespiratory responses. There was no difference (HR $P=0.731, \dot{V} \mathrm{O}_{2} P=0.798$, VT $P=0.071$ and $\left.\dot{V}_{\mathrm{E}} P=0.958\right)$ in the magnitude of peak cardiorespiratory changes induced by the different forms of ES exercise (Table 2). Similarly, the peak values during phase 3 (Table 2) were not affected by the exercise mode (HR $P=0.118$, $\dot{V}_{\mathrm{O}_{2}} P=0.846$, VT $P=0.843$ and $\left.\dot{V}_{\mathrm{E}} P=0.399\right)$. The average response (Table 3 ) values during phase 2 with FES cycling were not significantly different to those produced by isometric ES. The confidence intervals for the differences in the average responses are also displayed in Table 3

\section{DISCUSSION}

The primary purpose of this study was to compare the energy cost elicited by two modes of ES exercise-dynamic cycling and intermittent isometric contractions-in SCI subjects using the same ES protocol in both exercise modes. The principal finding of the study was that the $\dot{V}_{\mathrm{O}_{2}}$ for both modes of exercise was virtually identical. This similarity cannot be attributed to chance variations in characteristics between subjects, as the experimental design ensured that subjects were compared with themselves performing the two modes of exercise. In addition, the similarity of oxygen cost cannot be attributed to chance variations in the day-to-day response to exercise or to order effects, as in each of the two testing days a crossover was performed, comparing one mode of exercise with the other in reversed order. Along with similar oxygen cost, there were no significant differences in $\mathrm{HR}$ or ventilatory responses in comparing the two modes of exercise.

Consistent with the present findings, no significant difference in the energy cost of dynamic contractions and intermittent isometric contractions, using the same ES protocol, has been found in rat medial gastrocnemius muscle ${ }^{7}$ and human adductor pollicis. ${ }^{5}$ In contrast, Elder et al. ${ }^{8}$ found a significantly lower absolute energy cost, as assessed by pulmonary $\dot{V}_{\mathrm{O}_{2}}$, during ES dynamic bilateral knee extension compared with intermittent isometric contractions in ablebodied subjects. However, these authors also found that a significantly lower muscle mass was recruited during the isometric contractions compared with the dynamic contractions. When the authors calculated the increase in $\dot{V}_{\mathrm{O}_{2}}$ above resting levels relative to the amount of muscle mass recruited, they found no significant difference in the energy cost in comparing the two modes of exercise. The authors speculated that the differences in muscle recruitment may have been because of dynamic exercise bringing more nerve branches into the field of the ES compared with isometric actions. We suggest that, unlike the study by Elder et al., ${ }^{8}$ the absolute $\dot{V}_{\mathrm{O}_{2}}$ was similar in comparing dynamic with intermittent isometric actions in the present study because the level of muscle recruitment was similar in both exercise modes because of the higher current levels we used.

The current guidelines for physical activity for SCI recommend performing regular exercise each week. Unlike the general case for able-bodied individuals, in SCI patients one important issue is a potential limitation in the capacity to exercise at sufficiently high $\dot{V}_{\mathrm{O}_{2}}$ to elicit metabolic and cardiovascular health benefits.

ES of paralyzed muscles to produce cyclic leg movements allow SCI individuals to exercise at an intensity commensurate with the guidelines for able-bodied subjects in the range of light to moderate intensity. ${ }^{3}$ However, these modes of exercise are unlikely to benefit the majority of the SCI population as a practical means of performing life-long exercise on a weekly basis. The primary reason for this is the high equipment cost. $^{9-11}$ As an example, a commercially available hybrid cycling FES system available in the United Kingdom currently 
Table 2 Maximum values of cardiovascular measures achieved during the first 35 min (phase 2) and between 35 and 50 min (phase 3 ) of each exercise session (mean \pm s.d.; $N=8$ )

\begin{tabular}{|c|c|c|c|c|c|c|}
\hline & \multicolumn{3}{|c|}{ Session 1} & \multicolumn{3}{|c|}{ Session 2} \\
\hline & Phase 1 & Phase 2 & Phase 3 & Phase 1 & Phase 2 & Phase 3 \\
\hline & Rest & FES cycling $(35 \mathrm{~min})^{\mathrm{a}}$ & Isometric ES $(15 \mathrm{~min})^{\mathrm{a}}$ & Rest & Isometric ES $(35 \mathrm{~min})^{\mathrm{a}}$ & FES cycling $(15 \mathrm{~min})^{2}$ \\
\hline HR (b.p.m.) & $70 \pm 10$ & $107 \pm 21$ & $100 \pm 21$ & $65 \pm 10$ & $108 \pm 23$ & $107 \pm 23$ \\
\hline$\dot{\mathrm{V}}_{2}(\mathrm{ml} \mathrm{min}-1)$ & $231 \pm 57$ & $681 \pm 184$ & $585 \pm 180^{b}$ & $216 \pm 47$ & $674 \pm 195$ & $577 \pm 201^{b}$ \\
\hline VT BTPS (ml) & $479 \pm 51$ & $1214 \pm 268$ & $992 \pm 243^{b}$ & $472 \pm 63$ & $1278 \pm 260$ & $985 \pm 245^{b}$ \\
\hline$\dot{V}_{E}\left(I \min ^{-1}\right)$ & $7.8 \pm 1.3$ & $30.1 \pm 8.8$ & $23.8 \pm 8.0^{\mathrm{b}}$ & $7.1 \pm 1.2$ & $30.2 \pm 9.0$ & $25.8 \pm 11.8$ \\
\hline
\end{tabular}

Abbreviations: ES, electrical stimulation; FES, functional electrical stimulation; HR, heart rate. Indicates significant increase from rest for all measures.

bIndicates significant decrease from phase 2.

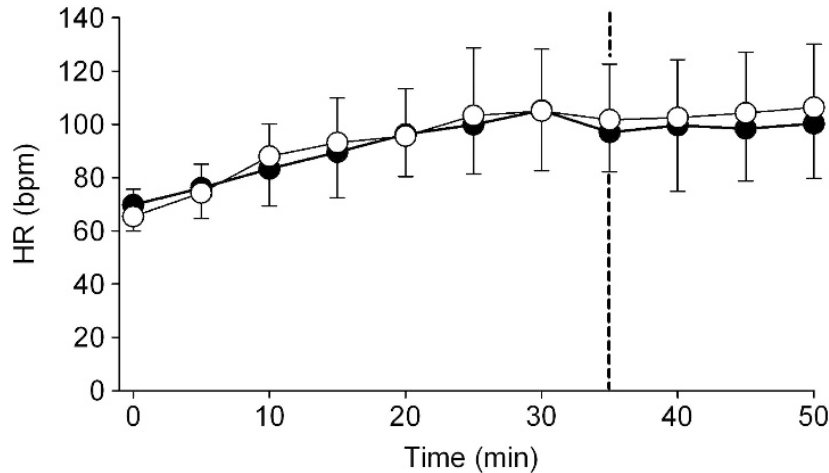

Figure $3 \mathrm{HR}$ versus time for sessions 1 and $2(N=8)$. Session $1(\bullet)$ and session $2(O)$. The dashed line represents the change between FES cycling and isometric ES (and vice versa). Data plotted as mean \pm s.d.; some error bars have been deleted for clarity.

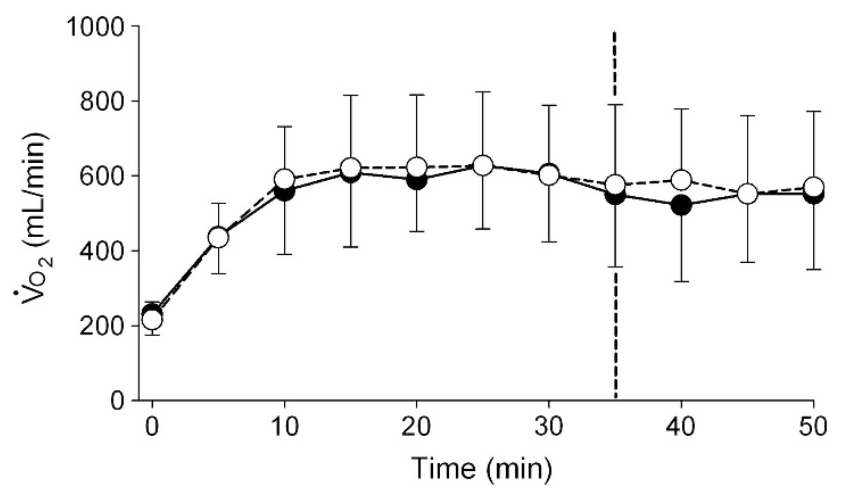

Figure 4 Oxygen consumption versus time for sessions 1 and $2(N=8)$. Session $1(0)$ and session $2(O)$. The dashed line represents the change between FES cycling and isometric ES (and vice versa). Data plotted as mean \pm s.d.; some error bars have been deleted for clarity.

retails for approximately $£ 9000 .{ }^{12}$ Such costs preclude individual purchase and home use by a substantial portion of the SCI population. Although it is conceivable that FES cycling equipment could be made available in public-access exercise facilities, again the high equipment costs and lack of specialist staff trained to support the use of such equipment form a barrier to the widespread access of these modes of exercise by SCI individuals. Additional barriers to

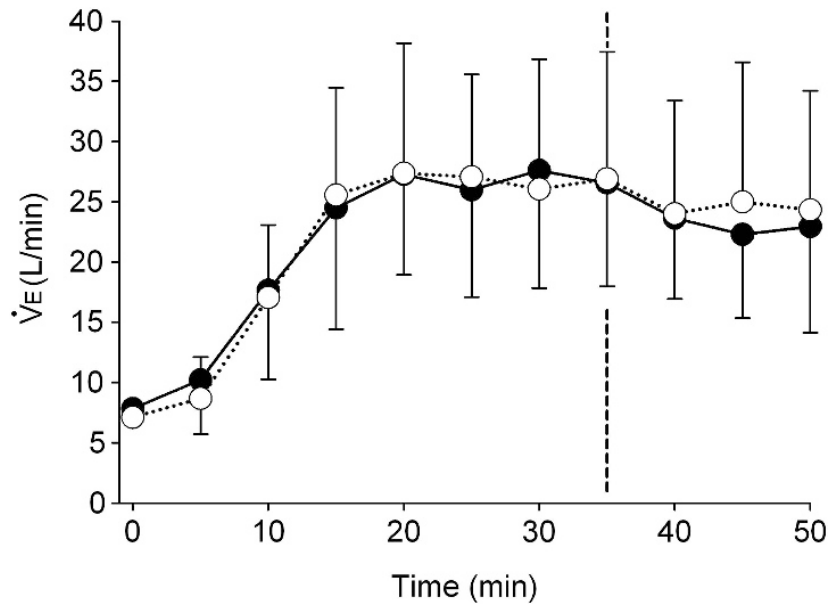

Figure 5 Ventilation versus time for sessions 1 and $2(N=8)$. Session $1(\bullet)$ and session $2(O)$. The dashed line represents the change between FES cycling and isometric ES (and vice versa). Data plotted as mean \pm s.d.; some error bars have been deleted for clarity.

Table 3 Average values of cardiovascular measures during the first $35 \mathrm{~min}$ (phase 2) of each exercise session (mean \pm s.d.; $N=8$ )

\begin{tabular}{|c|c|c|c|c|}
\hline & FES cycling ${ }^{a}$ & Isometric ES & $\begin{array}{l}\text { Statistical } \\
\text { comparison }\end{array}$ & $\begin{array}{c}\text { Confidence } \\
\text { intervals }^{b}\end{array}$ \\
\hline HR (b.p.m.) & $93 \pm 15$ & $95 \pm 17$ & $P=0.264$ & $-2 \pm 5$ \\
\hline$\dot{\mathrm{V}}_{2}\left(\mathrm{ml} \mathrm{min}^{-1}\right)$ & $534 \pm 128$ & $558 \pm 146$ & $P=0.451$ & $-24 \pm 137$ \\
\hline VT BTPS (ml) & $975 \pm 214$ & $1039 \pm 205$ & $P=0.193$ & $-64 \pm 210$ \\
\hline$\dot{V}_{E}\left(I m^{-1}\right)$ & $23.0 \pm 6.5$ & $23.8 \pm 6.7$ & $P=0.655$ & $-0.8 \pm 6.6$ \\
\hline
\end{tabular}

Abbreviations: ES, electrical stimulation; FES, functional electrical stimulation; HR, heart rate. andicates significant increase from rest for all measures. No significant differences were found between the increases induced by FES cycling and isometric ES.

${ }^{b}$ Column indicates confidence intervals for differences between means ( $80 \%$ power; $\alpha=0.05$ ), where a positive value indicates a higher FES cycling mean.

use of FES cycling exercise are the cumbersome nature of the equipment ${ }^{9,10}$ and the substantial time required for electrode placement before exercise, ${ }^{11}$ and the possible need for a period of pretraining to achieve a continuous cycling motion for an effective exercise session duration. ${ }^{2,9}$

The results of this study must be interpreted with caution because only a small $(n=8)$ sample size was used, increasing the chance of a 
type II error occurring; we cannot state that the two protocols (that is, FES cycling and isometric ES) elicit equal cardiorespiratory responses.

Nevertheless, although statistical power to find small or even medium differences was low, the nonsignificant differences between the exercise conditions that occurred in the context of highly significant differences between exercise and rest conditions, and between phase 2 and phase 3 conditions, suggest the design was suitable for detecting clinically important differences. This interpretation is supported by the confidence intervals calculated from the data that suggest the differences in cardiorespiratory response between the two modes are not clinically significant. For example, the maximum difference in $\dot{V}_{\mathrm{O}_{2}}$ is $<0.5$ METs (metabolic equivalent).

This study suggests that intermittent isometric ES can produce a clinically equivalent cardiorespiratory response as FES cycling exercise in SCI subjects. Isometric ES exercise has the potential to use relatively low-cost stimulation equipment and seating systems. We suggest that further research should investigate optimizing the stimulation parameters of intermittent isometric ES for SCI subjects, and to design affordable systems using this mode of exercise.

\section{DATA ARCHIVING}

There were no data to deposit.

\section{CONFLICT OF INTEREST}

The authors declare no conflict of interest.

\section{ACKNOWLEDGEMENTS}

Dr C Fornusek's salary was funded by the NSW Lifetime Care \& Support Authority.

1 Ginis KA, Hicks AL, Latimer AE, Warburton DE, Bourne C, Ditor DS et al. The development of evidence-informed physical activity guidelines for adults with spinal cord injury. Spinal Cord 2011; 49: 1088-1096.

2 Jacobs PL, Nash MS. Exercise recommendations for individuals with spinal cord injury. Sports Med 2004; 34: 727-751.

3 Hettinga DM, Andrews BJ. Oxygen consumption during functional electrical stimulation-assisted exercise in persons with spinal cord injury: implications for fitness and health. Sports Med 2008; 38: 825-838.

4 Fenn W. The relation between the work performed and the energy liberated in muscular contraction. J Physiol 1924; 58: 373-395.

5 Newham DJ, Jones DA, Turner DL, McIntyre D. The metabolic costs of different types of contractile activity of the human adductor pollicis muscle. J Physiol 1995; 488 (Pt 3), 815-819.

6 Fornusek C, Davis GM, Sinclair P, Milthorpe B. Development of an isokinetic functional electrical stimulation cycle ergometer. Neuromodulation 2004; 7: 56-64.

7 Beltman JG, van der Vliet MR, Sargeant AJ, de Haan A. Metabolic cost of lengthening, isometric and shortening contractions in maximally stimulated rat skeletal muscle. Acta Physiol Scand 2004; 182: 179-187.

8 Elder CP, Mahoney ET, Black CD, Slade JM, Dudley GA. Oxygen cost of dynamic or isometric exercise relative to recruited muscle mass. Dyn Med 2006; 5: 9.

9 Carty A, McCormack K, Coughlan GF, Crowe L, Caulfield B. Increased aerobic fitness after neuromuscular electrical stimulation training in adults with spinal cord injury. Arch Phys Med Rehabil 2012; 93: 790-795.

10 Wheeler GD, Andrews B, Lederer R, Davoodi R, Natho K, Weiss C et al. Functional electric stimulation-assisted rowing: increasing cardiovascular fitness through functional electric stimulation rowing training in persons with spinal cord injury. Arch Phys Med Rehabil 2002; 83: 1093-1099.

11 Bersch I, Frotzler A, Baumberger M. Functional electrical stimulation in spinal cord injury: clinical evidence versus daily practice. Biomed Tech 2013; 58 (Suppl).

12 What is FES cycling?, http://www.fescycling.com/pricing/. Anatomical Concepts (UK) Limited, 2013. 